\title{
Pedunculopontine Glutamatergic Neurons Provide a Novel Source of Feedforward Inhibition in the Striatum by Selectively Targeting Interneurons
}

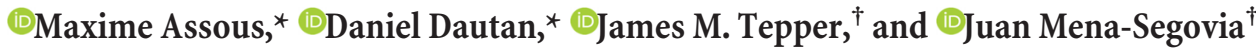 \\ Center for Molecular and Behavioral Neuroscience, Rutgers University, Newark, New Jersey 07102
}

The main excitatory inputs to the striatum arising from the cortex and the thalamus innervate both striatal spiny projection neurons and interneurons. These glutamatergic inputs to striatal GABAergic interneurons have been suggested to regulate the spike timing of striatal projection neurons via feedforward inhibition. Understanding how different excitatory inputs are integrated within the striatal circuitry and how they regulate striatal output is crucial for understanding basal ganglia function and related behaviors. Here, using VGLUT2 mice from both sexes, we report the existence of a glutamatergic projection from the mesencephalic locomotor region to the striatum that avoids the spiny neurons and selectively innervates interneurons. Specifically, optogenetic activation of glutamatergic axons from the pedunculopontine nucleus induced monosynaptic excitation in most recorded striatal cholinergic interneurons and GABAergic fastspiking interneurons. Optogenetic stimulation in awake head-fixed mice consistently induced an increase in the firing rate of putative cholinergic interneurons and fast-spiking interneurons. In contrast, this stimulation did not induce excitatory responses in spiny neurons but rather disynaptic inhibitory responses ex vivo and a decrease in their firing rate in vivo, suggesting a feedforward mechanism mediating the inhibition of spiny projection neurons through the selective activation of striatal interneurons. Furthermore, unilateral stimulation of pedunculopontine nucleus glutamatergic axons in the striatum induced ipsilateral head rotations consistent with the inhibition of striatal output neurons. Our results demonstrate the existence of a unique interneuron-specific midbrain glutamatergic input to the striatum that exclusively recruits feedforward inhibition mechanisms.

Key words: feedforward inhibition; glutamate; interneurons; mesencephalic locomotor region; pedunculopontine nucleus; striatum

Significance Statement

Glutamatergic inputs to the striatum have been shown to target both striatal projection neurons and interneurons and have been proposed to regulate spike timing of the projection neurons in part through feedforward inhibition. Here, we reveal the existence of a midbrain source of glutamatergic innervation to the striatum, originating in the pedunculopontine nucleus. Remarkably, this novel input selectively targets striatal interneurons, avoiding the projection neurons. Furthermore, we show that this selective innervation of interneurons can regulate the firing of the spiny projection neurons and inhibit the striatal output via feedforward inhibition. Together, our results describe a unique source of excitatory innervation to the striatum which selectively recruits feedforward inhibition of spiny neurons without any accompanying excitation.

\section{Introduction}

The striatum constitutes the main input structure of the basal ganglia. Its core physiological function is the integration of ex-

Received Nov. 13, 2018; revised March 1, 2019; accepted March 7, 2019.

Author contributions: M.A., D.D., J.M.T., and J.M.-S. designed research; M.A. and D.D. performed research; M.A. and D.D. analyzed data; M.A., D.D., J.M.T., and J.M.-S. wrote the first draft of the paper; M.A., D.D., J.M.T., and J.M.-S. edited the paper; M.A., D.D., J.M.T., and J.M.-S. wrote the paper.

This work was supported by National Institutes of Health Grant NS100824 to J.M.-S. and Grant NS034865 to J.M.T., National Alliance for Research on Schizophrenia and Depression Young Investigator Award to J.M.-S., and Rutgers University.

The authors declare no competing financial interests.

*M.A. and D.D. contributed equally to this work. trinsic inputs and their transmission to downstream basal ganglia structures. Cortex and thalamus comprise two of its major excitatory inputs (Kemp and Powell, 1971; Albin et al., 1989; DeLong, 1990; Silberberg and Bolam, 2015), innervating both spiny projection neurons (SPNs) and interneurons. Most striatal

†J.M.T. and J.M.-S. contributed equally to this work.

Correspondence should be addressed to Juan Mena-Segovia at juan.mena@rutgers.edu or James M. Tepper at jtepper@newark.rutgers.edu.

D. Dautan's present address: Department of Neuroscience and Brain Technologies, Genetics of Cognition laboratory, Istituto Italiano di Tecnologia, via Morego, 30, 16163 Genova, Italy.

https://doi.org/10.1523/JNEUROSCI.2913-18.2019

Copyright $\odot 2019$ the authors 
GABAergic interneurons inhibit SPNs monosynaptically (Koós and Tepper, 1999; Gittis et al., 2010; Ibáñez-Sandoval et al., 2010, 2011; Szydlowski et al., 2013; Straub et al., 2016; but see Assous et al., 2018), and cholinergic interneurons (CINs) disynaptically through nicotinic activation of striatal GABAergic interneurons (English et al., 2011; Faust et al., 2016), thus suggesting that striatal interneurons are in a position to modulate the activity of SPNs through feedforward inhibition. Notably, glutamatergic afferents make stronger connections onto interneurons than principal cells in many regions, supporting the idea that interneurons can acutely shape network activity through feedforward inhibitory mechanisms (Parthasarathy and Graybiel, 1997; Ramanathan et al., 2002; Gabernet et al., 2005; Mallet et al., 2005; Cruikshank et al., 2007; Isaacson and Scanziani, 2011). So far, the most studied form of feedforward inhibition in striatum is through cortical activation of fast-spiking interneurons (FSIs) (Koós and Tepper, 1999; Mallet et al., 2005; Martiros et al., 2018; Owen et al., 2018); but given the prominent excitatory innervation originating in the thalamus and other subcortical structures (Kemp and Powell, 1971; Albin et al., 1989; DeLong, 1990; Pan et al., 2010; Wall et al., 2013; Silberberg and Bolam, 2015; Klug et al., 2018), it is likely that they also contribute to shaping the activity of striatal interneurons (Assous and Tepper, 2019) and, consequently, driving feedforward inhibition. Understanding how different excitatory inputs are integrated within striatal circuitry and how they regulate striatal output is crucial for understanding basal ganglia-related function and behavior.

We recently described a novel pathway that originates in the pedunculopontine nucleus (PPN) and whose activation modulates the activity of striatal circuits by increasing the discharge of cholinergic interneurons and inhibiting the activity of SPNs (Dautan et al., 2018). While these effects were associated with cholinergic neurons of the PPN, retrograde tracing studies revealed that a large proportion of striatal-projecting neurons in the PPN were noncholinergic (Dautan et al., 2014). Glutamatergic neurons constitute the largest neuronal population in the PPN (Wang and Morales, 2009) and innervate different components of the basal ganglia, including midbrain dopaminergic neurons (Ros et al., 2010; Galtieri et al., 2017) and the striatum (Klug et al., 2018). Furthermore, PPN glutamatergic neurons have recently been implicated in the regulation of motor activity as a downstream target of the basal ganglia (Roseberry et al., 2016; Caggiano et al., 2018), suggesting that their bidirectional connectivity with basal ganglia nuclei may underlie their role in motor behavior.

Using a combination of anatomical and electrophysiological approaches, here we report the existence of a glutamatergic projection to the striatum originating in the PPN that selectively innervates interneurons and produces feedforward inhibition of SPNs. Activation of this pathway induces ipsiversive head movements. Our results thus demonstrate the existence of a midbrain excitatory projection whose activation modulates striatal network activity strictly through feedforward inhibition of SPNs.

\section{Materials and Methods}

All procedures used in this study were performed in agreement with the National Institutes of Health Guide to the care and use of laboratory animals and with the approval of the Rutgers University Institutional Animal Care and Use Committee (Newark). Male and female WT and VGLUT2-Cre (hemizygotic; The Jackson Laboratory, \#016963) mice were bred, housed in groups of up to 4 per cage, and maintained on a $12 \mathrm{~h}$ light cycle (7:00 A.M. to 7:00 PM) with ad libitum access to food and water. All experiments used mice of both sexes.
Tracing experiments. Adult male and female WT and VGLUT2-Cre weighting between 15 and $20 \mathrm{~g}$ were anesthetized with isoflurane $(1.5 \%$ $2.5 \%$, delivered with $\mathrm{O}_{2}, 1 \mathrm{ml} / \mathrm{min}$ ) and placed in a stereotaxic frame. Bupivacaine was used as a local anesthetic at the site of the incision. Animals were then injected with an AAVr-CAG-Td-tomato (200 nl; gift from the Janelia Research Institute and the laboratory of Dr. J. Dudman) or Fluorogold (400 nl, 2\% in saline for WT mice; Fluorochrome) or AAVr-Flex-TdTomato ( $400 \mathrm{nl}$; Addgene, \#28306) in the dorsal striatum $(2 \times 200 \mathrm{nl}$ in the dorsomedial and the dorsolateral striatum; anteroposterior $1.0 \mathrm{~mm}$; mediolateral $2.0 \mathrm{~mm}$; dorsoventral 3.5/3.0/2.5 mm from dura) to visualize retrogradely labeled neurons. To analyze the topography of the striatal projections, we injected AAV-Flex-YFP (50 nl, University of North Carolina Vector Core) or AAV-DIO-GFP-2AmRuby-synaptophysin (Virus Core, Stanford University). Following 2 weeks of recovery, animals were transcardially perfused and the brain tissue processed for immunocytochemistry. For all experiments, animals where the injection site was not constrained to dorsal striatum were discarded from further analyses.

Immunocytochemistry and immunofluorescence. Following behavioral, electrophysiological, or anatomical experiments, mice were anesthetized with an overdose of pentobarbital ( $200 \mathrm{mg} / \mathrm{kg}$, i.p.) and then transcardially perfused with $0.05 \mathrm{M}$ PBS followed by ice-cold $4 \%$ PFA in PBS (50 $\mathrm{ml}$ ). Brains were prepared for either coronal or parasagittal sections (50 $\mu \mathrm{m})$ in PBS using a vibratome (VT1200S, Leica Microsystems). For ex vivo experiments, following recording, slices containing the biocytinfilled neurons were transferred to a $4 \%$ PFA solution for $48 \mathrm{~h}$ before being processed. Slices were then transferred to a blocking solution (10\% normal donkey serum in $1 \%$ Triton X-100 in PBS) for $1 \mathrm{~h}$. Sections were then washed and incubated overnight with an antibody against ChAT (Abcam, AB144P, made in goat, 1/500), parvalbumin (Synaptic Systems, 195004, made in guinea pig, 1/500) or GFP (Invitrogen, A21311, made in rabbit, 1/1000). Following several washes, sections were incubated for $4 \mathrm{~h}$ in CY5-conjugated donkey antibody (anti-goat, Jackson ImmunoResearch Laboratories, 705-175-147, 1/1000) and Alexa-488-conjugated donkey antibody (anti-rabbit, Jackson ImmunoResearch Laboratories, 711-545-152, 1/1000). All sections were also processed for biocytin using a CY3-congugated streptavidin solution in PBS containing $0.03 \%$ Triton. Sections resulting from tracer injections, in vivo electrophysiology, and behavioral experiments were processed with antibodies against GFP (as above) or mCherry (anti-rabbit, Abcam, AB167453, made in rabbit, $1 / 1000)$. High-resolution images were captured using a confocal microscope (Olympus Fluoview FV1200). Images were analyzed using ImageJ software.

Ex vivo brain slice recordings. VGLUT2-Cre mice were injected with AAV-Flex-ChR2-GFP (200 nl, University of North Carolina Vector Core) in the PPN (anteroposterior -4.5 , mediolateral 1.25, dorsoventral $3.3 \mathrm{~mm}$ ). Approximately 6 weeks after virus injection, mice were deeply anesthetized with an intraperitoneal injection of $80 \mathrm{mg} / \mathrm{kg}$ ketamine $/ 20$ $\mathrm{mg} / \mathrm{kg}$ xylazine, and perfused transcardially with an ice-cold $\mathrm{N}$-methyl D-glucamine (NMDG)-based solution containing the following (in mM): 103.0 NMDG, $2.5 \mathrm{KCl}, 1.2 \mathrm{NaH}_{2} \mathrm{PO}_{4}, 30.0 \mathrm{NaHCO}_{3}, 20.0$ HEPES, 10.0 glucose, $101.0 \mathrm{HCl}, 10.0 \mathrm{MgSO}_{4}, 2.0$ thiourea, 3.0 sodium pyruvate, $12.0 \mathrm{~N}$-acetyl cysteine, $0.5 \mathrm{CaCl}_{2}$ (saturated with $95 \% \mathrm{O}_{2}$ and $5 \% \mathrm{CO}_{2}$, $\mathrm{pH}$ 7.2-7.4). After decapitation, the brain was quickly removed into a beaker containing ice-cold oxygenated NMDG-based solution before slicing. Oblique parahorizontal sections, $300 \mu \mathrm{m}$ in thickness, were cut in the same medium using a Vibratome 3000 . Sections were immediately transferred to an oxygenated NMDG-based solution at $35^{\circ} \mathrm{C}$ for $5 \mathrm{~min}$, after which they were transferred to oxygenated normal Ringer's solution at $25^{\circ} \mathrm{C}$ until used.

The recording chamber was constantly perfused $(2-4 \mathrm{ml} / \mathrm{min})$ with oxygenated Ringer's solution at $32^{\circ} \mathrm{C}-34^{\circ} \mathrm{C}$. Drugs were applied in the perfusion medium and were dissolved freshly each day in Ringer's solution. Slices were initially visualized under epifluorescence illumination with a digital frame transfer camera (Cooke SensiCam) mounted on an Olympus BX50-WI epifluorescence microscope with a $40 \times$ long working distance water-immersion lens to visualize the transduction field in the striatum. Visualization was then switched to infrared-differential interference contrast microscopy for the actual patching of the neuron. 
Micropipettes for whole-cell recording were constructed from $1.2 \mathrm{~mm}$ outer diameter borosilicate pipettes on a Narishige PP-83 vertical puller. The standard internal solution for whole-cell current-clamp recording was as follows (in mM): $130 \mathrm{~K}$-gluconate, $10 \mathrm{KCl}, 2 \mathrm{MgCl}_{2}, 10 \mathrm{HEPES}, 4$ $\mathrm{Na}_{2}$ ATP, $0.4 \mathrm{Na}_{2} \mathrm{GTP}$, pH 7.3. Biocytin (0.2\%, Sigma-Aldrich) was also added to the internal solution to allow description of the neuronal morphology of recorded neurons and post hoc identification with immunocytochemistry. To visualize disynaptic inhibitory events in SPNs in voltage clamp, we used a CsCl-based internal solution containing the following (in mM): $125 \mathrm{CsCl}$, 0.1 EGTA, 10 HEPES, $2 \mathrm{MgCl}_{2}, 4 \mathrm{Na}_{2} \mathrm{ATP}$, and $0.4 \mathrm{Na}_{2} \mathrm{GTP}$. This solution also contained $0.2 \%$ (wt) AlexaFluor-594 or biocytin (Sigma-Aldrich) to verify the identity of SPNs online morphologically. These pipettes had a DC impedance of 3-5 $\mathrm{M} \Omega$. Membrane currents and potentials were recorded using an Axoclamp 700B amplifier (Molecular Devices) and digitized at $20-40 \mathrm{kHz}$ with a CED Micro 1401 Mk II and a PC running signal, version 5 (Cambridge Electronic Design). Optogenetic stimulation consisted of a $5 \mathrm{~ms}$ duration blue light pulses (440 nm LED, Mouser Electronics). Sweeps were run at $20 \mathrm{~s}$ intervals.

The onset latency was defined as the time between the beginning of the optogenetic stimulation pulse and the onset of the postsynaptic response. The time to peak was determined as the time between the beginning of the stimulation pulse and the peak of the postsynaptic response.

In vivo head-fixed recordings. Six weeks after AAV-Flex-ChR2-GFP (200 nl, University of North Carolina Vector Core) injection into the PPN, animals underwent a second surgery where an implant for head fixation was cemented to the skull around a $\sim 1.5 \mathrm{~mm} \times 1.5 \mathrm{~mm}$ craniotomy centered on the dorsal striatum (anteroposterior 0.7 , mediolateral 1.8). The craniotomy was kept protected using biocompatible silicone (Smooth-On, \#0718). Mice were habituated to the head-fixed setup on top of a treadmill. Briefly, mice were exposed to the apparatus and to head fixation in periods of increasing length and with interruptions when mice had free access to food and water in their home cage. Recordings began following habituation of 3-5 consecutive days.

On the day of the recording, a silicone probe (OA1x16-50-177, Neuronexus) was slowly lowered using a micromanipulator (Scientifica), and the striatum was scanned for single-unit activity. Extracellular signals were digitized and amplified using an Intan chip (Intan Technologies, RHD2216) and recorded as wide band signals using Intan software (RHD2000). When the signal from an individual neuron was acquired and exhibited a signal-to-noise ratio of at least 3:1, a baseline of 3-5 min was recorded. Next, between 60 and 240 optogenetic pulses $(20 \mathrm{~ms}, 0.5$ $\mathrm{Hz}, 3 \mathrm{~mW}$ ) were delivered through the optic fiber under the control of a TTL generator (Arduino Uno) and triggered by the recording software. After each recording, the probe was moved in a random direction for 200-400 $\mu \mathrm{m}$ (anteroposterior or mediolateral) to avoid multiple recordings of the same neuron. Recorded signals were converted to smr files (Spike2, Cambridge Electronic Design) using a custom MATLAB script. Signals were then bandpass filtered between 500 and $5000 \mathrm{~Hz}$ and sorted using the wavemark option in Spike2. Only neurons that showed a confidence interval of $>95 \%$ of the action potential (AP) principal component analyses were considered as individual neurons and used for further analyses. The firing rate before ( $5 \mathrm{~s})$, during ( $50 \mathrm{~ms}$ for interneurons and $100 \mathrm{~ms}$ for SPNs), and after (5 s) was determined using a peristimulus time histogram of the average interspike interval (iFr). Percentage changes of $>10 \%$ were considered as responding neurons. To calculate changes in activity, the iFr was $z$ scored based on the $5 \mathrm{~s}$ before the stimulation. Statistically significant effects were considered changes of $\geq \pm 2$ SD of the $z$ score. The putative nature of recorded neurons was estimated based on previous experiments and was classified using basal firing activity, the coefficient of variation, and AP waveform. Neurons presenting a basal firing rate between 0 and $2 \mathrm{~Hz}$ and a half-width AP duration between 1 and $2 \mathrm{~ms}$ were considered as putative SPNs (pSPNs); putative FSI (pFSIs) have a shorter half-width $\mathrm{AP}(<1 \mathrm{~ms})$ and a higher firing rate $(2-16 \mathrm{~Hz})$, whereas putative CINs (pCINs) have a higher firing rate than pSPNs $(>2 \mathrm{~Hz})$ and a wider half-width AP than FSIs.

Motor behavior. Six weeks after AAV-Flex-ChR2-GFP (200 nl, University of North Carolina Vector Core) injection in the PPN, VGLUT2-Cre mice and WT controls were implanted with an optic fiber $(200 \mu \mathrm{m}, 0.5$ NA, Thorlabs) at the level of the dorsal striatum (anteroposterior 0.75, mediolateral 1.75, dorsoventral 2.0), maintained in position with an anchor screw located in the contralateral striatum and all covered in dental cement. Following 5-7 d of recovery, animals were placed in a twocompartment box (total of $80 \mathrm{~cm} \times 40 \mathrm{~cm} \times 40 \mathrm{~cm}$ ) for $30 \mathrm{~min}$. Each compartment was randomly attributed to an experimental condition: (1) no stimulation or (2) optogenetic stimulation. Animal locomotion and head-body rotation (angular velocity) were monitored using tracking software (Anymaze, Stoelting). All stimuli had a duration of $1 \mathrm{~s}$ with an interstimulus interval of $9 \mathrm{~s}$. A laser pulse $(10 \mathrm{~Hz}, 20 \mathrm{~ms})$ was delivered through the cannula under the control of a shutter (SH1, Thorlabs) triggered by TTL pulses generated by the animal tracking interface (Ami1). To account for the possible effect of the sound of the shutter, the nonstimulation condition triggered a similar shutter opening protocol but with the laser turned off. The head-body rotation was obtained by calculating the absolute head-body angle $1 \mathrm{~s}$ before the opening of the shutter to $1 \mathrm{~s}$ during stimulation. The variation of the head-body angular velocity (Delta) was defined as a change from the angle formed by the head-body axis versus the body-tail axis.

Experimental design and statistics. For the anatomical experiments, a total of 12 mice were used as follows: 3 WT mice for Fluorogold injections, 3 WT mice for AAV-retro-CAG-TdTomato injections, 3 VGLUT2-Cre mice for AAV-retro-Flex-Tdtomato injections, and 3 VGLUT2-Cre mice for AAV-DIO-YFP injections. For ex vivo electrophysiology, a total of 15 VGLUT2-Cre mice were used. A paired $t$ test was used to compare the responses during optogenetic stimulation and after drug perfusion. For in vivo electrophysiology, a total of 6 VGLUT2-Cre mice were used for extracellular recordings. More than 300 neurons were recorded, and 37 were selected for further analysis based on signal-tonoise ratio $>3$, AP variation $<1 \%$, and interspike interval $>2 \mathrm{~ms}$. Analysis of the firing rate changes (expressed as percentage change) was defined by comparing the recordings during the laser stimulation (50 or $100 \mathrm{~ms}$ ) to the recordings immediately before the stimulation (baseline). Firing rates were converted to $z$ scores. One-way or two-way ANOVAs were used to compare the percentage change for all groups and stimulation protocols. For behavioral experiments, a total of 6 VGLUT2-cre and 5 WT mice were used. Parameters of distance traveled, average speed, and head-body/tail-body axes were obtained using Anymaze software, and the effect of the optogenetic stimulation was evaluated by comparing these parameters before each stimulation (baseline) and during the stimulation ( $1 \mathrm{~s}$ ). A two-way ANOVA (group $\times$ stimulation) was used to compare the percentage change and the $z$ score variation. Data were excluded in those cases where the injection site was out of target, as defined by post hoc histological analysis. For in vivo and ex vivo recordings, cells that were not classified either by their electrophysiological or neurochemical (i.e., immunohistochemistry) properties were not further analyzed. All statistical tests were run using Prism or StataSE.

\section{Results}

\section{PPN glutamatergic neurons innervate the striatum}

We previously demonstrated in rats, using anterograde and retrograde anatomical tracings, that cholinergic neurons of the PPN innervate the striatum (Dautan et al., 2014). From the retrogradely labeled neurons, $\sim 60 \%-70 \%$ of the PPN neurons were immunopositive for ChAT, suggesting the existence of noncholinergic neurons of the PPN that project to striatum. To determine the proportions of cholinergic and noncholinergic PPN neurons that project to the striatum in the mouse, we first injected a retrograde tracer (Fluorogold) into the striatum of WT mice (C57BL/6, The Jackson Laboratory; $n=3$ ). We found that $64.5 \%$ of PPN-labeled neurons were ChAT-negative (Fig. $1 A-D$ ). Similar results were obtained after the injection of a retrograde AAV that transduced a fluorescent reporter (200 nl, AAV-retroCAG-TdTomato) into the striatum of WT mice (C57BL/6, The Jackson Laboratory; $n=3$ ). We found that $>60 \%$ of retrogradely labeled PPN neurons were ChAT-immunonegative (Fig. 1E-H). To determine whether striatal-projecting, noncholinergic neurons were glutamatergic, we injected an AAV retro-Flex-tdTomato 

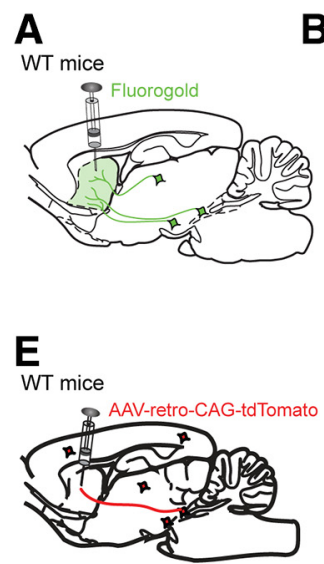

VGLUT2-Cre

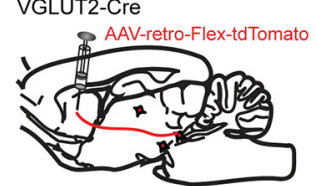

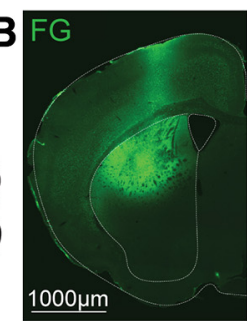
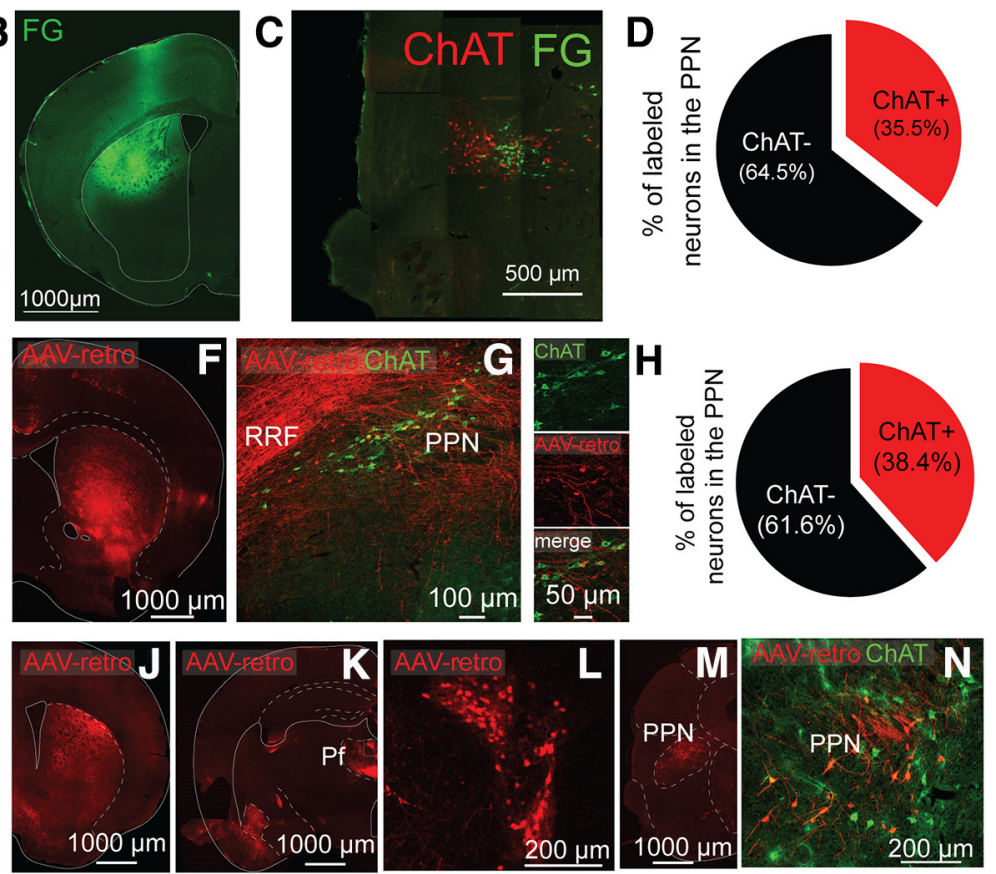

Figure 1. Striatal retrograde tracing reveals cholinergic and glutamatergic projections originating in the PPN. $A-D$, Injection of fluorogold into the striatum reveals $C$ ChAT ${ }^{+}$and $C h A T^{-}$neurons in the PPN. $\boldsymbol{A}$, Experimental design. $\boldsymbol{B}$, Injection site in striatum. C, Retrogradely labeled neurons in PPN delimited by ChAT immunostaining (red). $\boldsymbol{D}$, Quantification of ChAT $^{+} / \mathrm{ChAT}^{-}$retrogradely $^{-}$ labeled neurons. $\boldsymbol{E}-\boldsymbol{H}$, Striatal injection of AAV retro reveals similar proportions of $\mathrm{ChAT}^{+}$and $\mathrm{ChAT}^{-}$neurons in the PPN. $\boldsymbol{E}$, Experimental design. $\boldsymbol{F}$, Striatal injection site. $\mathbf{G}$, Retrogradely labeled neurons in the PPN (red) combined with ChAT immunocytochemistry (green). $\boldsymbol{H}$, Percentage of retrogradely labeled neurons in the PPN expressing ChAT. Similar numbers of retrogradely labeled $\mathrm{ChAT}^{+}$and $\mathrm{ChAT}^{-}$neurons were found using fluorogold (D) or AAV-retro $(\boldsymbol{H}) . \boldsymbol{I}$, Cre-dependent AAV-retro-td-tomato virus was injected in the striatum of VGLUT2-Cre mice.J, Striatal injection site. $\boldsymbol{K}-\boldsymbol{L}$, Retrogradely labeled neurons in the parafascicular nucleus of the thalamus and the PPN $(\boldsymbol{M}, \boldsymbol{N})$, as delimited by ChAT immunostaining $(\boldsymbol{N})$.
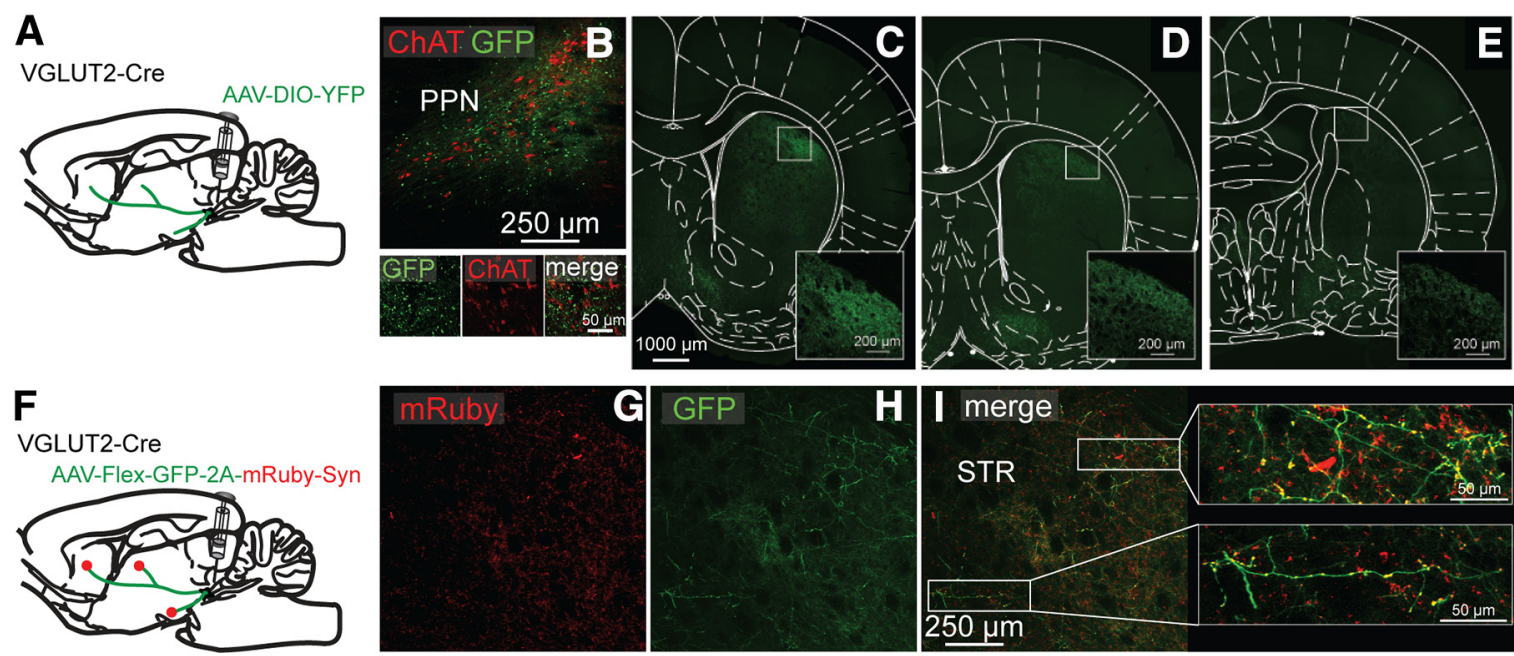

Figure 2. Anterograde anatomical tracing of PPN glutamatergic neurons reveals synaptic contacts in the striatum. $\boldsymbol{A}$, Injection of a Cre-dependent YFP virus in the PPN of VGLUT2-Cre mice. $\boldsymbol{B}$, Injection site in the PPN delimited by ChAT immunostaining (red). $\boldsymbol{C}-\boldsymbol{E}$, YFP-expressing PPN axons in the striatum at three different anteroposterior levels ( $\boldsymbol{C}$, anteroposterior $1 \mathrm{~mm}$; $\boldsymbol{D}$, anteroposterior $0.6 \mathrm{~mm}$; and $\boldsymbol{E}$, anteroposterior $-0.34 \mathrm{~mm}$ from bregma). $\boldsymbol{F}$, Injection of a Cre-dependent AAV-DIO-GFP-2A-mRuby-synaptophysin in the PPN of VGLUT2-Cre mice. $\boldsymbol{G}, \boldsymbol{H}, \mathrm{mRuby}$ and GFP axon labeling in the striatum. I, Higher magnification of mRuby-positive synaptic terminals in the striatum (red).

into the striatum of VGLUT2-Cre mice. We detected tdTomatolabeled neurons in the PPN, as defined by the ChAT staining outline (Fig. $1 I-N$ ), indicating that PPN glutamatergic neurons innervate the striatum. Furthermore, we detected retrogradely labeled PPN neurons both ipsilateral and contralateral to the striatal injection, suggesting that these projections are bilateral (data not shown). Only $\sim 4 \%$ of PPN VGLUT2-positive neurons that project to the striatum were also ChAT-positive, in agreement with a previous report (Wang and Morales, 2009; and data not shown). Next, we examined the distribution of PPN gluta- matergic axons in the striatum by transducing them with a fluorescent reporter injected into the PPN (AAV2-DIO-YFP, $50 \mathrm{nl}$, VGLUT2-Cre mice, $n=3$; Fig. $2 A$ ). We detected the presence of YFP-positive axons across large areas of the both the dorsal and ventral striatum (Fig. $2 C-E$ ). Within the striatum, we observed a dorsoventral as well as anteroposterior gradient of these projections where the dorsal part (close to the corpus callosum) is more innervated than the ventral part, and the anterior part is more densely innervated than the posterior part. Finally, conditional labeling of the presynaptic protein synaptophysin in PPN gluta- 
A VGLUT2-Cre

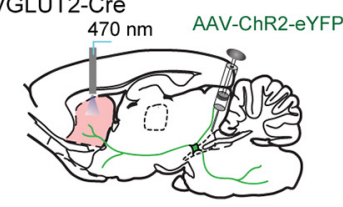

B

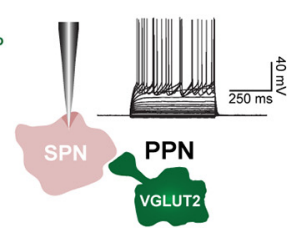

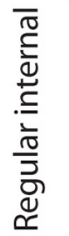

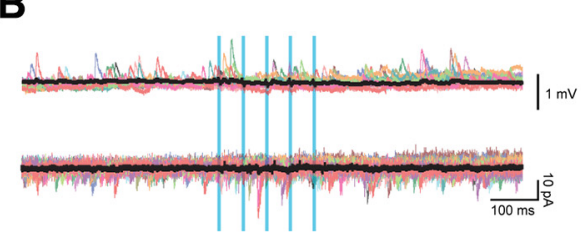

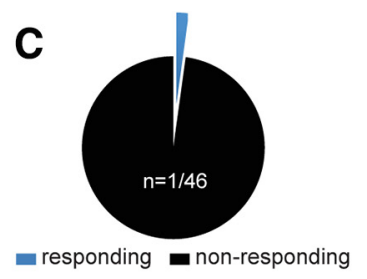

responding $\boldsymbol{n}$ non-responding
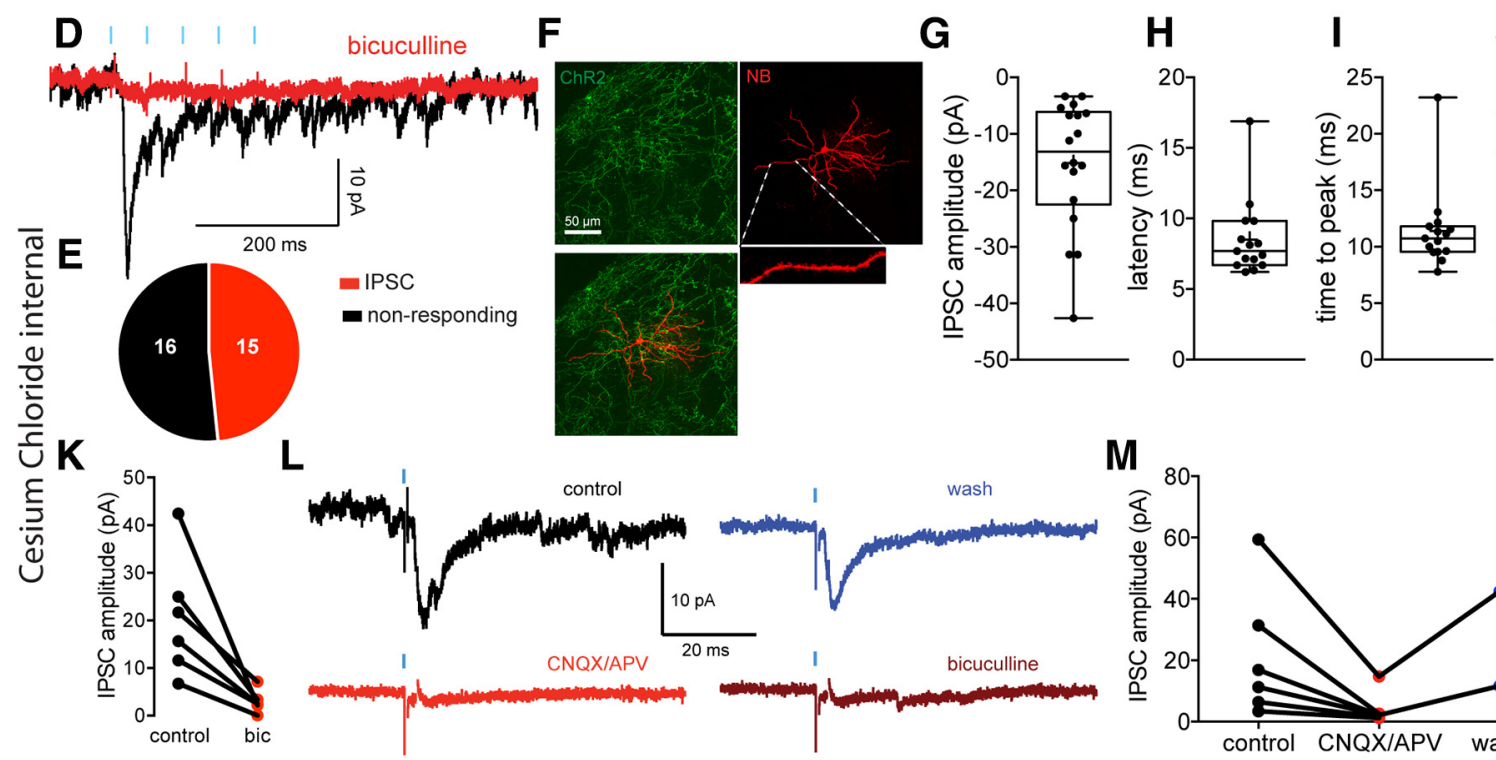

$\mathbf{J}$

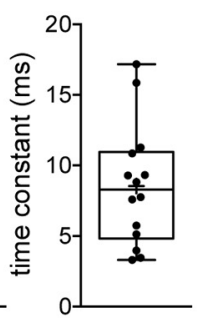

M

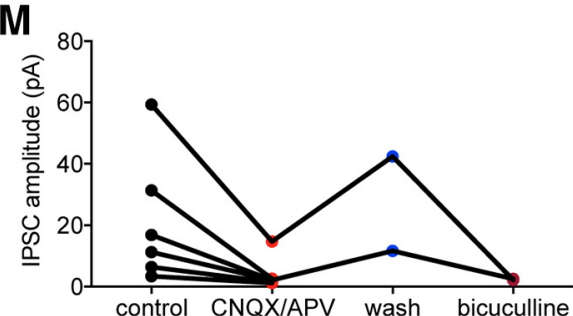

Figure 3. Lack of innervation of SPNs by PPN glutamatergic neurons. $A$, Injection of a Cre-dependent ChR2-eYFP virus in the PPN of VGLUT2-Cre mice, and responses to somatic current injection in a representative SPN. B, Current-clamp (top) and voltage clamp (bottom, $V_{h}=-70 \mathrm{mV}$ ) recordings of an SPN illustrating the lack of response to PPN optogenetic stimulation. Color traces represent individual trials. Black traces represent average. $C$, Pie chart of the percentage of SPNs responding to optogenetic stimulation of PPN glutamatergic striatal axons. The vast majority of recorded SPNs do not respond $(97.8 \%, n=45$ of 46$)$. $\boldsymbol{D}$, Voltage-clamp responses of a SPN $\left(V_{\mathrm{h}}=-70 \mathrm{mV}\right)$ to optogenetic stimulation of PPN glutamatergic striatal axons $(5$ pulses, $20 \mathrm{~Hz})$, using high $\mathrm{CSCl}^{-}$internal (125 mM) to amplify inhibitory responses that are $\mathrm{GABA}_{\mathrm{A}}$-mediated, as they are blocked by bicuculline $(10 \mu \mathrm{M})$. $\boldsymbol{E}$, Summary of SPN inhibitory responses. $\boldsymbol{F}$, Biocytin-filled SPNs (red) surrounded by ChR2-expressing PPN glutamatergic terminals (green). $\mathbf{G}-\boldsymbol{J}$, Box plots of the amplitude $(\boldsymbol{G})$, latency $(\boldsymbol{H})$, time to peak $(\boldsymbol{I})$, and time constant $(\boldsymbol{J})$ of the inhibitory responses. The longer latency, consistent with a disynaptic effect. $\boldsymbol{K}$, Quantification of bicuculline pharmacology (GABAA receptor antagonist, $10 \mu \mathrm{m}, n=6$ ) on the IPSC amplitude. $\boldsymbol{L}$, Voltage-clamp response of a SPN to stimulation of PPN axons (black, control). The response can be blocked by glutamate receptor antagonists (CNQX/APV, $10 \mu \mathrm{M}$, red, $n=6)$ as well as bicuculline (10 $\mu \mathrm{m}$, brown) after wash (blue). $M$, Quantification of glutamatergic and $G A B A_{A}$ pharmacology on the PPN-evoked response in SPNs. Box plots represent the minimum, maximum interquartile range, the mean, and median. Blue bars indicate optical stimulation.

matergic axons (AAV-DIO-GFP-2A-mRuby-synaptophysin in VGLUT2-Cre mice) revealed the presence of synaptophysinpositive axon terminals throughout the entire extent of the striatum (Fig. 2G-J). These results demonstrate the existence of a glutamatergic projection originated in the PPN that forms synaptic contacts across large extents of the striatum.

PPN glutamatergic neurons do not directly innervate SPNs To identify the striatal synaptic target(s) of PPN glutamatergic neurons, a floxed ChR2 virus (200 nl; AAV2-DIO-ChR2, $n=15$ mice) was injected into the PPN of VGLUT2-Cre mice and whole-cell recordings were obtained from striatal neurons in acute brain slices. SPNs were identified in brain slices based on their well-established intrinsic electrophysiological properties (Fig. $3 A$ ) and post hoc after streptavidin labeling of their densely spinous dendritic arborization. Surprisingly, essentially none of the recorded SPNs in the transduction field exhibited the expected monosynaptic excitatory responses to optogenetic stimulation of PPN glutamatergic striatal terminals $\left(V_{\mathrm{h}}=-70 \mathrm{mV}\right.$; $n=45$ of 46 not responding; $97.8 \%$ Fig. $3 B, C$ ), and the single SPN that responded exhibited only a very small inward current $(\sim 4 \mathrm{pA})$. Those results demonstrate that PPN glutamatergic neurons do not monosynaptically innervate SPNs.
We then examined responses of SPNs to activation of PPN glutamatergic inputs using an internal solution containing 125 $\mathrm{mm} \mathrm{CsCl}$ to enhance detection of GABAergic IPSCs. In contrast to the results described above, under these conditions, we observed that, in $48.38 \%$ of the recorded SPNs, optogenetic stimulation of PPN glutamatergic inputs elicited IPSCs ( $n=15$ of 31 ; $15.16 \pm 2.661 \mathrm{pA}$; Fig. $3 D-M$ ). These IPSCs were mediated by $\mathrm{GABA}_{\mathrm{A}}$ receptors as they were blocked by bicuculline $(10 \mu \mathrm{M}$, $n=6$; two-tailed paired $t$ test, $p=0.0172$; time constant: $8.515 \pm$ $1.142 \mathrm{~ms}, n=14$; Fig. $3 D-M)$ and exhibited unusually long latencies ( $8.459 \pm 0.698 \mathrm{~ms}$; Fig. $3 \mathrm{H}, \mathrm{I} ; n=15)$ implying mediation by polysynaptic pathways. The IPSCs were also blocked by bath application of AMPA/NMDA glutamate receptor antagonists (Fig. $3 L, M ; n=6$ ), which confirmed the polysynaptic nature of the response. These results suggest that activation of PPN glutamatergic axons in the striatum induced feedforward inhibition of SPNs.

PPN glutamatergic neurons innervate striatal cholinergic and fast-spiking interneurons

We recorded from a number of interneuron types, including large numbers of CINs and FSIs and smaller numbers of LTS and TH interneurons (Tepper et al., 2018). CINs were identified in 


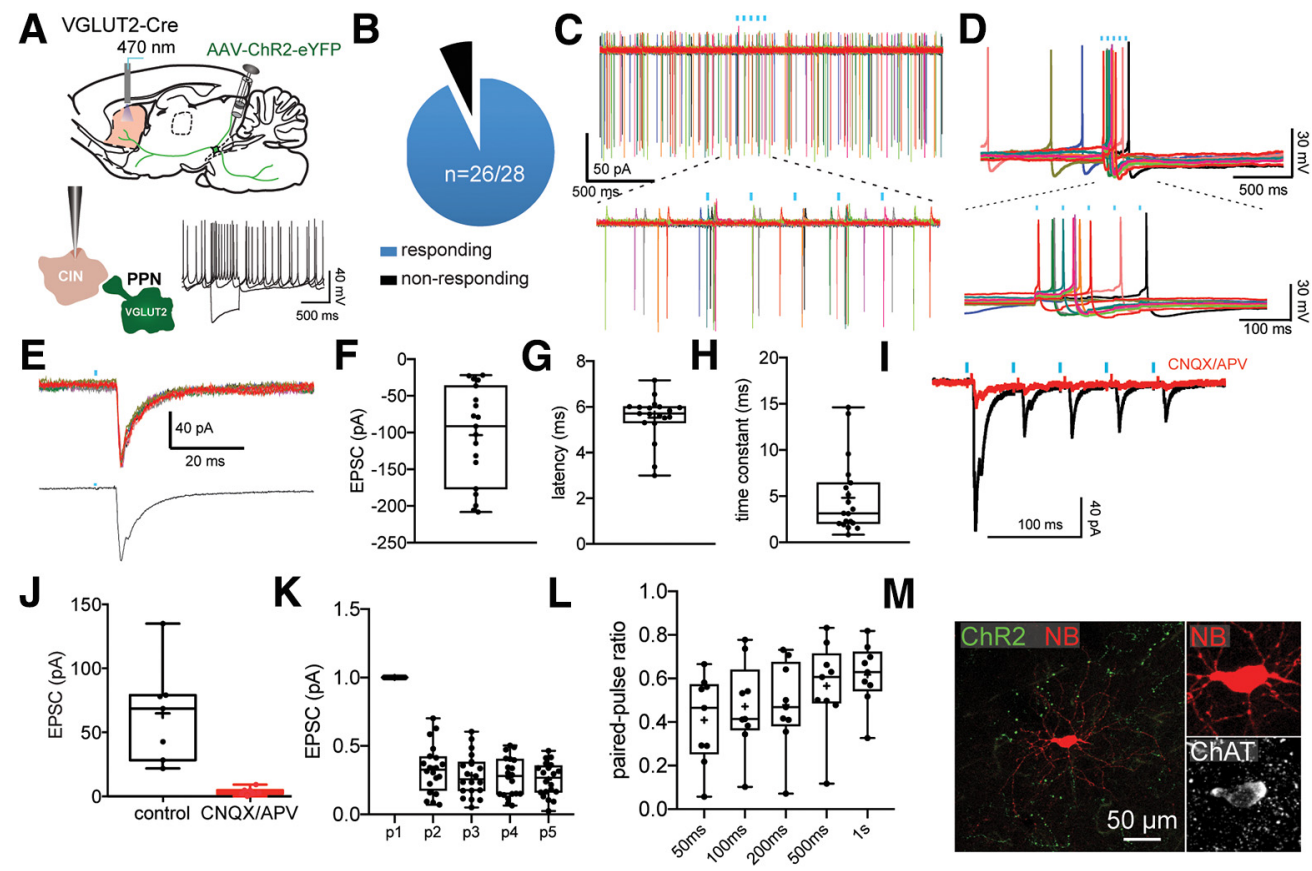

Figure 4. Monosynaptic innervation of CINs by PPN glutamatergic neurons. $A$, Responses to somatic current injection of a representative CIN after transduction with a Cre-dependent ChR2-eYFP virus into the PPN of VGLUT2-Cre mouse. $\boldsymbol{B}$, Pie chart of the percentage of CINs responding to optogenetic stimulation of PPN glutamatergic striatal axons. $\boldsymbol{C}$, Cell-attached recording of a spontaneously active CIN. Bottom, The response to optogenetic stimulation is enlarged. $\boldsymbol{D}$, Current-clamp recording of a spontaneously active CIN. Bottom, The response to optogenetic stimulation is enlarged. E, EPSC evoked by optogenetic stimulation of PPN glutamatergic striatal axons in a CINs. Top, Individual traces. Bottom, Average. F-H, Box plots showing the EPSC size, latency, and time constant. $\boldsymbol{I}$, The EPSC induced by optogenetic stimulation is blocked by bath application of glutamate receptor antagonists (CNQX and APV, $10 \mu \mathrm{M}$ ). Quantification in box plot in $\boldsymbol{J}$. $\boldsymbol{K}$, Box plot quantifying the response to a train of 5 stimuli $(20 \mathrm{~Hz})$. Results are expressed as a PPR to the first EPSC. L, Box plot quantifying the response to a train of 2 stimuli (PPR) at different interstimulus intervals. Results are expressed as a PPRs. $\boldsymbol{M}$, CIN filled with biocytin (red) surrounded by ChR2 terminals (green) colocalizing with ChAT immunostaining (white). Blue bars indicate optical stimulation.

slices by their large cell body size, their unique intrinsic properties (e.g., spontaneous tonic firing, long duration $\mathrm{AP}, \mathrm{I}_{\mathrm{h}}$ sag current; Fig. 4A), and post hoc following immunostaining for ChAT (Fig. $4 M)$. In contrast to SPNs, the vast majority of CINs responded to the optogenetic stimulation of PPN glutamatergic axons $(n=26$ of 28; 92.9\%; Fig. 4B). The stimulation ( 5 pulses, $20 \mathrm{~Hz}$ ) evoked large EPSPs and AP firing in CINs both in cell-attached and in whole-cell current clamp (Fig. 4C,D). Whole-cell voltage-clamp recordings $\left(V_{\mathrm{h}}=-70 \mathrm{mV}\right)$ revealed that the EPSC is relatively large $(103.48 \pm 15.23 \mathrm{pA} ; n=19)$ and exhibits strong short-term depression (p1: 89.33 \pm 17.59; p2: $27.74 \pm 8.28 ; \mathrm{p} 3: 26.2 \pm 6.76$; p4: $23.43 \pm 6.53$; p5: $22.82 \pm 5.71$ pA; $n=19$; Fig. $4 E-L)$. We also measured changes in the amplitude of a test EPSC produced by a preceding, conditioning terminal volley and calculated the paired-pulse ratio ( $\mathrm{PPR})$. We used a range of different interstimulus intervals $(50,100,200,500$, and $1000 \mathrm{~ms})$. Our results confirm the depressing nature of the PPN glutamatergic synapses onto CINs, which persist even at longer interstimulus intervals (results are expressed as a ratio of the first EPSC, $\mathrm{PPR}_{50 \mathrm{~ms}}: 0.41 \pm$ 0.067; $\mathrm{PPR}_{100 \mathrm{~ms}}: 0.47 \pm 0.068 \mathrm{PPR}_{200 \mathrm{~ms}}: 0.48 \pm 0.068 ; \mathrm{PPR}_{500 \mathrm{~ms}}$ : $0.57 \pm 0.069 ; \mathrm{PPR}_{1000 \mathrm{~ms}}: 0.62 \pm 0.047 ;$ Fig. $\left.4 L\right)$. Consistent with a monosynaptic activation of CINs by PPN glutamatergic terminals, the excitatory responses exhibit a short-onset latency $(5.52 \pm 0.23 \mathrm{~ms}$; Fig. $4 G)$ and are blocked by AMPA/NMDA glutamate receptor antagonists (CNQX and APV, respectively; 10 $\mu \mathrm{M} ; 94.23 \pm 2.01 \%$ reduction in EPSC size; paired $t$ test; $p=$ $0.0051 ; n=7$; Fig. $4 I, J$ ). While this latency is longer compared with some optogenetic studies (Cruikshank et al., 2007; Brill et al., 2016), it is consistent with the latencies of monosynaptic activation by extrinsic inputs previously reported (Saunders et al., 2016; Assous et al., 2017; Klug et al., 2018).
Next, we measured the response of FSIs to optogenetic stimulation of PPN glutamatergic neurons. FSIs were identified based on their intrinsic electrophysiological properties (low input resistance, high rheobase current, fast AP firing at depolarized current steps; Fig. 5A) and the expression of parvalbumin (Fig. $5 J$ ). Similar to CINs, the vast majority of FSIs ( $n=7$ of $9 ; 77.8 \%$; Fig. $5 B$ ) responded to the stimulation with EPSCs $(n=6 ; 134.5 \pm 30.9$ pA; Fig. 5C,E) that exhibited a strong short-term depression (5 pulses, $20 \mathrm{~Hz}$; p1: $155.7 \pm 29.02$; p2: $60.16 \pm 15.76$; p3: $80.67 \pm$ 27.46; p4: $58.2 \pm 21.66$; p5: $72.13 \pm 22.4$ pA; $n=4$; Fig. $5 I$ ). Consistent with a monosynaptic innervation of striatal FSIs by PPN glutamatergic neurons, EPSCs/EPSPs exhibited a shortonset latency ( $5.49 \pm 0.26 \mathrm{~ms}$; Fig. $5 F-H)$ and were blocked by glutamate receptor antagonists (CNQX and APV $10 \mu \mathrm{M} ; n=5$; $93.95 \pm 0.58 \%$ reduction; paired $t$ test; $p=0.0006$; Fig. $5 C, D)$.

Additionally, we recorded the light-evoked response of a limited number of two other striatal GABAergic interneuron populations, the $\mathrm{TH}$ expressing GABAergic interneurons and NPY-expressing low-threshold spike (LTS) interneurons that were identified based on their intrinsic electrophysiological properties (Kawaguchi, 1993; Ibáñez-Sandoval et al., 2010). Both interneurons showed suprathreshold excitatory responses to the optogenetic stimulation similar to those observed in CINs and FSIs (data not shown). Together, these results show that PPN glutamatergic neurons selectively innervate every subtype of striatal interneuron tested thus far.

\section{PPN glutamatergic neurons modulate striatal output}

To identify the impact of excitatory transmission arising in the PPN on striatal function, we tested the effects of optogenetic stimulation of PPN glutamatergic axons on striatal neurons in 

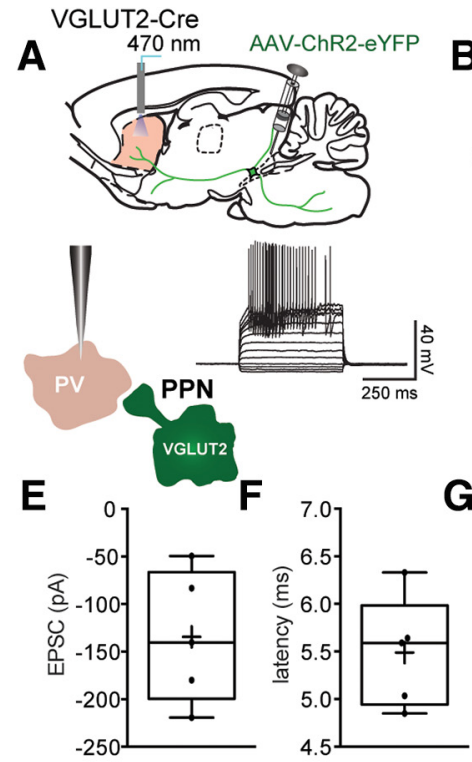
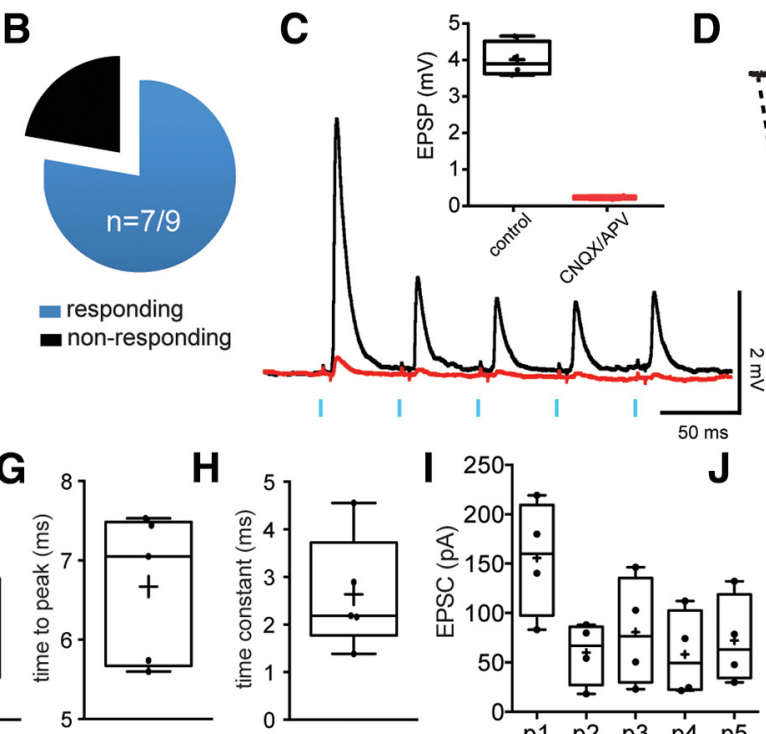

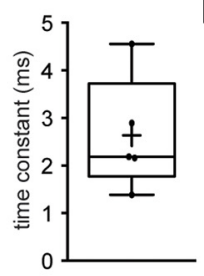

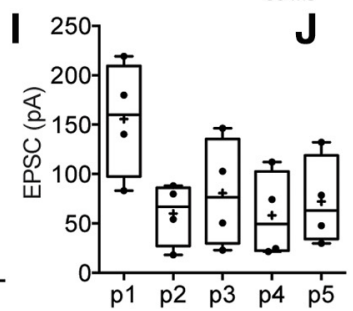

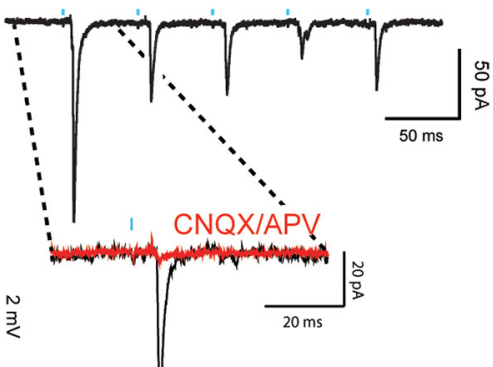

Figure 5. Monosynaptic innervation of FSIs by PPN glutamatergic neurons. $\boldsymbol{A}$, Responses to somatic current injection in a representative FSI after transduction with a Cre-dependent ChR2-eYFP virus into the PPN of a VGLUT2-Cre mouse. B, Pie chart of the percentage of FSIs responding to the optogenetic stimulation of PPN glutamatergic striatal axons. C, Current-clamp recording of optogenetic stimulation of an FSI. The stimulation ( 5 pulses, $20 \mathrm{~Hz}$ ) evoked EPSPs are blocked by CNQX and APV (10 $\mu \mathrm{M}$ ), quantification in inset box plot. $D$, EPSCs in an FSI evoked by optogenetic stimulation of PPN glutamatergic axons. Inset, EPSC is blocked by CNQX and APV as in $\boldsymbol{C}$. $\boldsymbol{E}-\boldsymbol{H}$, Box plots representing the EPSC size $(\boldsymbol{E})$, latency $(\boldsymbol{F})$, time to peak $(\boldsymbol{G})$, and time constant $(\boldsymbol{H})$. $\boldsymbol{I}$, Box plots of the responses to a train of 5 stimuli at $20 \mathrm{~Hz}$ show the depressing nature of the EPSC. J, FSI filled with biocytin (red) surrounded by ChR2 terminals (green) colocalized with parvalbumin immunostaining (white). Blue bars indicate optical stimulation.

vivo. First, to determine whether PPN-driven feedforward inhibitory mechanisms occurred in vivo, we recorded the electrophysiological responses of striatal neurons following the local optogenetic stimulation of PPN glutamatergic axons in awake, head-fixed mice using multicontact electrodes (Fig. 6A). We recorded extracellularly 37 single units that were clustered as pSPNs, pFSIs, or pCINs, based on their basal firing rate, AP duration, and coefficient of variation (see Materials and Methods; $n=6$ mice). Stimulation of PPN glutamatergic axons produced a significant decrease in the firing rate of $\mathrm{pSPNs}(n=11$ of 17 ; basal firing rate: $0.61 \pm 0.089 \mathrm{~Hz}$; firing rate during stimulation: $0.47 \pm$ $0.074 \mathrm{~Hz})\left(n=17\right.$; paired $t$ test, $\left.t_{(33)}=-8.40, p=0.000001\right)$, whose latency $(71.714 \pm 5.53 \mathrm{~ms})$ suggested the involvement of polysynaptic pathways (Fig. $6 B, E-G$ ). Consistent with the results obtained in ex vivo whole-cell recordings, we detected an increase in the firing rate of pFSIs ( $n=6$ of $6 ; 100 \%$; basal: $5.96 \pm 0.91 \mathrm{~Hz}$, stimulation: $22.54 \pm 5.14 \mathrm{~Hz}$; paired $t$ test, $t_{(11)}=3.72, p=$ $0.0034)$ and pCINs $(n=11$ of $14 ; 78.6 \%$; basal: $5.64 \pm 0.72 \mathrm{~Hz}$, stimulation: $8.8 \pm 1.19 ; \mathrm{Hz}$ paired $t$ test, $t_{(27)}=8.0091, p=$ $0.00001)$, both with a shorter latency than SPNs $(9.83 \pm 0.6 \mathrm{~ms}$ and $9.07 \pm 0.22 \mathrm{~ms}$, respectively) and consistent with monosynaptic activation (Fig. $6 C-G$ ). Additionally, we also detected longlatency increases in the firing rate of both pFSIs and pCINs, which we attributed to the involvement of polysynaptic pathways, but they were not further analyzed (Fig. 6E).

Next, to determine the impact of PPN-driven, feedforward inhibition of SPNs on striatal functions, we tested the motor effects of the unilateral stimulation of PPN glutamatergic axons in freely moving mice. For this purpose, PPN neurons in VGLUT2-Cre mice were transduced with ChR2 as before, and an optic fiber was chronically implanted into the right dorsal striatum $(n=6)$. A control group consisted of WT mice receiving the same injections and implantations $(n=5)$. Optogenetic stimulation ( 1 s every $9 \mathrm{~s}, 10 \mathrm{~Hz}, 20 \mathrm{~ms}$ pulses, $3 \mathrm{~mW}$; Fig. $7 A-C$ ) had no significant effect on the distance traveled (two-way ANOVA: group effect: $F_{(1,18)}=0.81, p=0.3813$; Fig. $\left.7 D-F\right)$ but produced ipsiversive head rotations, an effect that has recently been associated with the optogenetic inhibition of the direct and indirect striatal projection pathways (Tecuapetla et al., 2014) (comparison between the angles formed by the head-body axis with the body-tail axis; angle: $8.91 \pm 14.83^{\circ}$ before vs $57.97 \pm 20.59^{\circ}$ after PPN axon stimulation; in the control group: $-6.26 \pm 3.95^{\circ}$ before vs $-7.94 \pm 2.54^{\circ}$ after stimulation; two-way ANOVA: stimulation $\times$ group, stimulation effect, $F_{(1,21)}=2.37, p=0.1399$; group effect, $F_{(1,21)}=1.13, p=0.3004$; interaction effect, $F_{(2,21)}$ $=3.74, p=0.0426$; Bonferroni post hoc test: PPNstim vs PPNnostim $=0.0096$; ctrlstim vs ctrlnostim $=1.0$; Figure $7 G-J$ ). These results suggest that, in the behaving mouse, activation of PPN glutamatergic axons in the striatum is able to inhibit SPNs and consequently reduce the striatal output.

\section{Discussion}

Our results in this study demonstrate the existence of an excitatory glutamatergic projection that originates in the PPN and modulates striatal neuronal activity. Using ex vivo recordings, we show that this projection selectively targets multiple subtypes of striatal interneurons and generates feedforward inhibition in SPNs. Using in vivo extracellular recordings, we demonstrate that striatal interneurons are activated following the stimulation of PPN glutamatergic axons, and SPNs show a long-latency decrease in firing rate. Last, in behaving animals, we show that unilateral optogenetic activation of this pathway induces ipsilateral head rotations, consistent with inhibition of SPNs. Our results then reveal a unique mechanism by which midbrain glutamatergic projections selectively recruit striatal interneurons resulting in suppression of striatal output.

One of the critical findings of this study is the demonstration of a novel source of feedforward inhibition to SPNs originating in glutamatergic neurons of the PPN. Interneuron-mediated feedforward inhibition has been shown to be a key mechanism for 
A
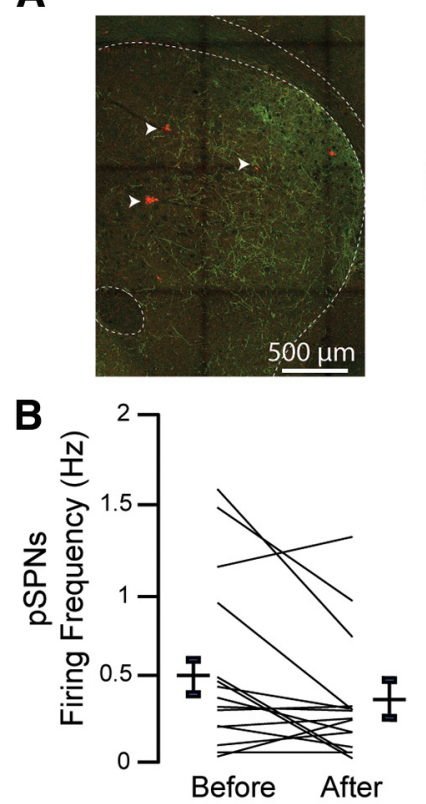
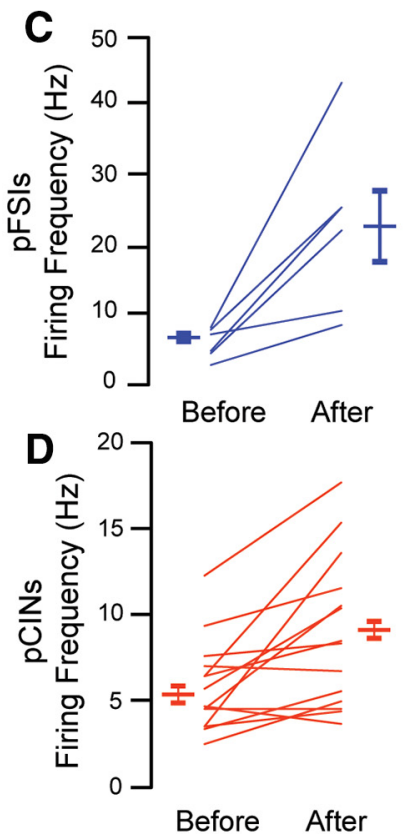

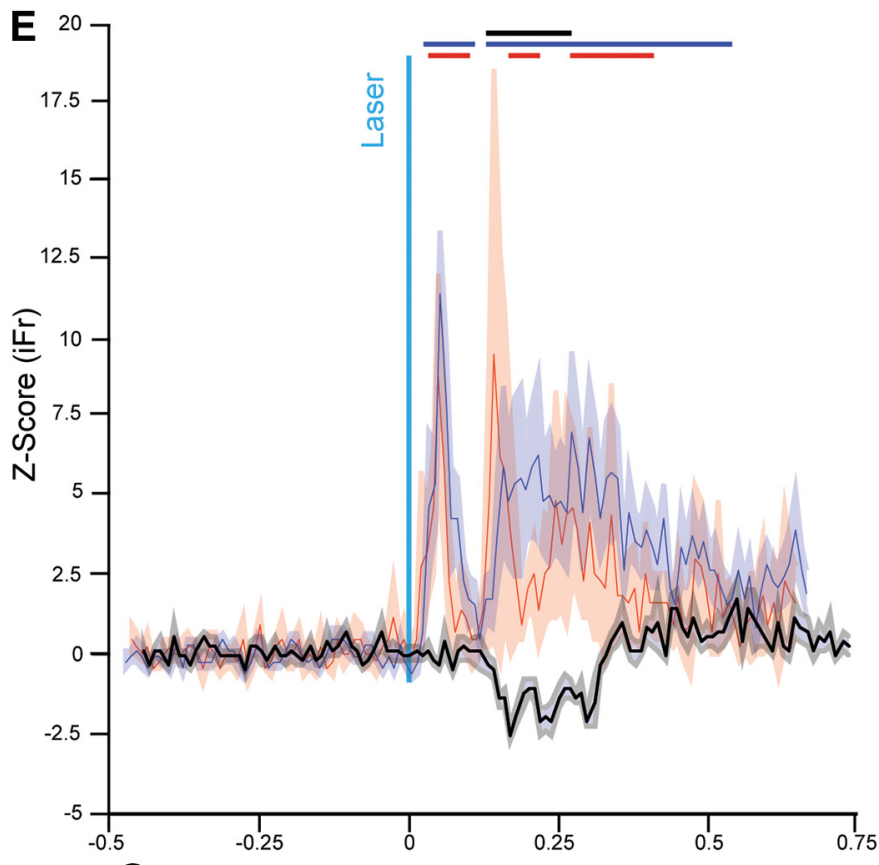

G

F
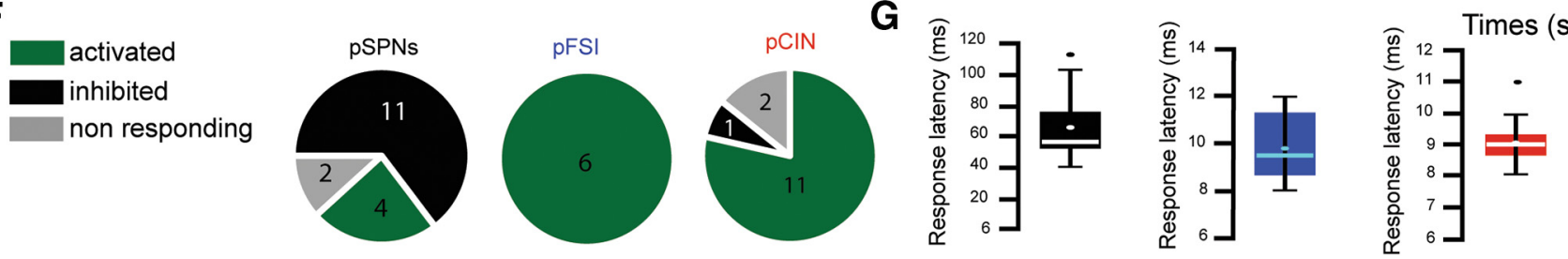

Figure 6. Stimulation of PPN glutamatergic axons in the striatum in vivo activates interneurons and induces feedforward inhibition of pSPNs. $\boldsymbol{A}$, Fluorescent image showing YFP-positive PPN axons (green) in the striatum in close proximity to the recording electrode tracks (white arrows). $\boldsymbol{B}-\boldsymbol{G}$, Changes in firing rate of pSPNs ( $\boldsymbol{B}$ ), pFSIs (C), and pCINs (D) before and after stimulation. $\boldsymbol{E}$, Normalized $z$ score of pSPNs (black), pFSI (blue), and pCINs (red) before and after stimulation. Bars indicate \pm 2 SD of the $z$ score for each group. $\boldsymbol{F}$, Proportion of responding neurons ( $\geq 2$ SD from z score) identified as pSPN (left), pFSI (middle), or pCINs (right) following stimulation of PPN axons. G, Response latencies for pSPN (left, black), pFSI (middle, blue), or pCINs (right, red).

regulating striatal activity. In structures, such as the cortex or hippocampus, local inhibition is mediated by interneurons via two types of regulatory mechanisms: feedback and feedforward inhibition (Isaacson and Scanziani, 2011). Both forms of inhibition are involved in regulating spike timing and network gain, controlling temporal integration and expanding the dynamic range of responses (Gabernet et al., 2005; Cruikshank et al., 2007; Pouille et al., 2009; Atallah et al., 2012; Lee et al., 2012; Moyer et al., 2014; Owen et al., 2018). In contrast, in the striatum, interneurons regulate spike timing of SPNs only through feedforward inhibition due to the GABAergic nature of the principal cells (Tepper et al., 2008). Here, following optogenetic activation of PPN glutamatergic axons, we identified excitatory responses in CINs and FSIs as well as in a limited number of TH and LTS GABAergic interneurons, thus suggesting that the PPN is able to activate most, if not all, striatal interneurons. An alternative scenario is possible in which there is selective innervation of interneuron subtypes, such as those described for the thalamus (NPY-expressing NGF and LTS interneurons) (Assous et al., 2017); however, there are no data to support this idea. Because FSIs, TH, and LTS interneurons are GABAergic and directly innervate SPNs (Koós and Tepper, 1999; Gittis et al., 2010; IbáñezSandoval et al., 2010, 2011; Straub et al., 2016), and because CINs induce potent disynaptic inhibition of SPNs through activation of several populations of striatal GABAergic interneurons (English et al., 2011; Nelson et al., 2014; Faust et al., 2016), our results thus reveal the existence of a source of excitation arising in the midbrain that is selective for interneurons and induces a longlatency polysynaptic inhibition of SPNs without concurrent excitation. The question of a selective feedforward inhibition of either direct- or indirect-pathway SPNs was not tested. However, the interneurons targeted by the PPN glutamatergic neurons (FSIs, LTS, TH as well as the GABAergic interneurons responsible for the disynaptic inhibition of SPNs after CINs activation) have been shown to target both direct and indirect pathway SPNs (Gittis et al., 2010; Ibáñez-Sandoval et al., 2010, 2011; English et al., 2011; Xenias et al., 2015), suggesting that both SPNs populations are subject to this feedforward inhibition pathway originating from the PPN. Interestingly, striatal interneurons are also interconnected to each other in a highly specific manner; and therefore, disinhibitory mechanisms may also play a role in this midbrain modulation (e.g., TH interneuron-mediated inhibition of LTS interneurons) (Assous et al., 2017; Assous and Tepper, 2019).

The glutamatergic inputs to striatal CINs have been shown to originate mostly from the intralaminar thalamic nuclei (predominantly expressing VGLUT2) and, more moderately, from different areas of the cortex (predominantly expressing VGLUT1). Thalamic inputs to striatal CINs have been shown to modulate goal-directed learning following changes in action-outcome contingencies (Bradfield et al., 2013), and are also suggested to participate in the typical pause-burst response of CINs following the presentation of a salient stimulus (Matsumoto et al., 2001; Goldberg and Reynolds, 2011; Schulz and Reynolds, 2013). Electro- 
A

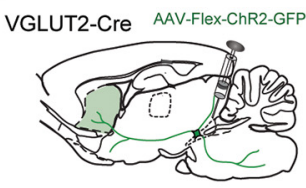

Ipsilateral - Controlateral

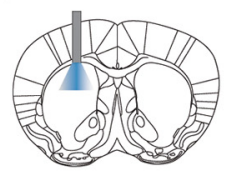

B

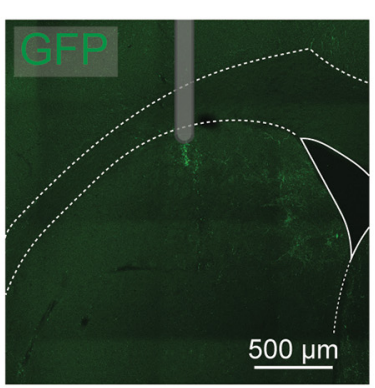

$\mathbf{F}$

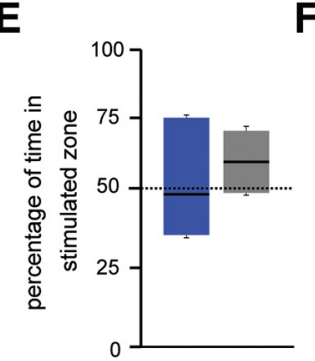

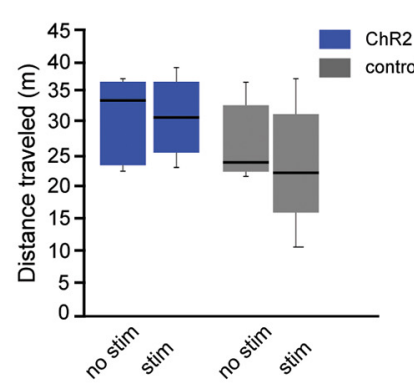

C

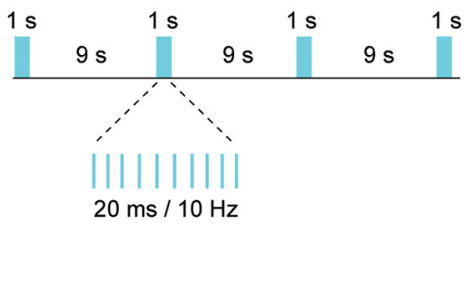

G

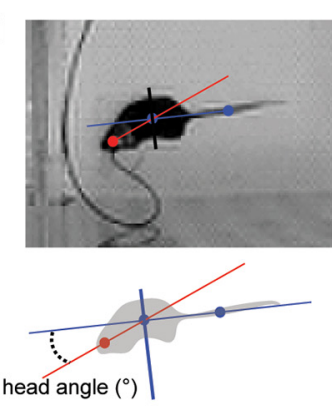

D

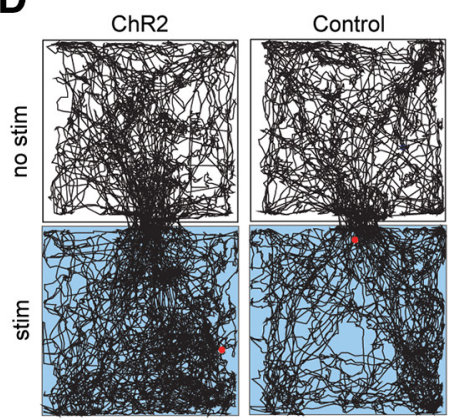

H no-stimulation trial stimulation trial

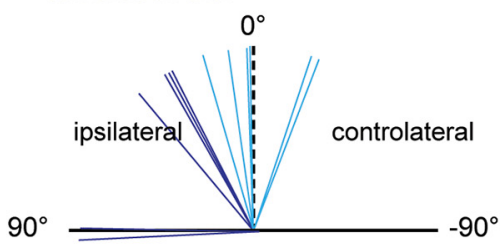

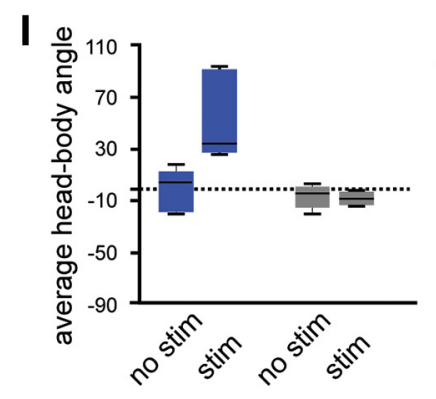

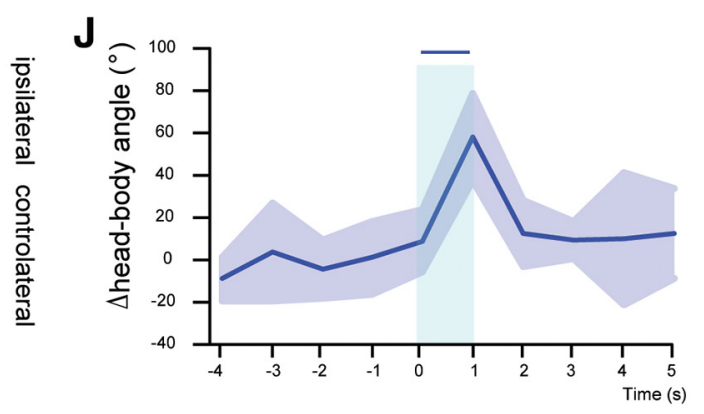

Figure 7. Stimulation of PPN glutamatergic axons in the striatum induces ipsilateral head movements. $\boldsymbol{A}$, Injection of AAV2-Flex-ChR2-GFP into the PPN of VGLUT2-Cre or WT mice. $\boldsymbol{B}$, Location of the optic fiber in the dorsal striatum. C, Schematic of stimulation parameters. D, Representative open field tracking of VGLUT2-Cre mice transduced with ChR2 in the PPN (left), compared to a control mouse (right). Top, No stimulation ("no stim"). Bottom, Laser stimulation ("stim"). E, Percentage of time spent in the stimulated compartment. Blue represents stimulation compartment. Gray represents no stimulation compartment. $\boldsymbol{F}$, Distance traveled by the animals with or without stimulation of the PPN or controls (gray). Stimulation of PPN does not change the distance traveled by the animals in the open field ( 30 min session). $\boldsymbol{G}$, Representation of the head-body (red) and tail-body (blue) axes. $\boldsymbol{H}$, Variation ( $\delta$ ) of angular velocity in the nonstimulated trials (light blue) versus the stimulated trials (dark blue). I, Angular velocity in nonstimulated (no stim) or stimulated (stim) trials. J, Change of the angular velocity over time.

physiologically, this multiphasic pattern of firing of CINs has been attributed, in part, to the facilitating nature of the thalamostriatal synapses onto CINs (Ding et al., 2010; Doig et al., 2014). Here, we show that the amplitude and the suprathreshold nature of the responses to PPN glutamatergic axons in the striatum are comparable with those originating in the thalamus (Ding et al., 2010; Assous et al., 2017; for review, see Smith et al., 2004, 2014). Furthermore, the nature of the response of striatal interneurons (FSIs and CINs) to the PPN optogenetic stimulation is also multiphasic, consisting of a short latency increase (most likely monosynaptic) and a second increase in firing rate after a longer delay. This late response likely involves polysynaptic extrastriatal relays, such as the thalamus or the cortex, structures that are also innervated by the PPN glutamatergic axons and provide innervation to striatal neurons. However, the short-term plasticity of the PPN to CINs synapses is strongly depressing and contrasts with that mediated by the synapses originating from the thalamus, thus suggesting a different effect, and perhaps a different role, of thalamic and PPN glutamatergic axons on CINs.

In this study, we also reveal that striatal-projecting midbrain PPN glutamatergic neurons are embedded in a region that has been functionally defined as the mesencephalic locomotor region (for review, see Mena-Segovia and Bolam, 2017). Together with the cuneiform nucleus, activation of mesencephalic locomotor region excitatory pathways has been suggested to modulate locomotor speed and gait (Roseberry et al., 2016; Caggiano et al., 2018). Recent studies (Josset et al., 2018), including our own unpublished data, however, have reported that the optogenetic activation of PPN glutamatergic neurons, rather than inducing an increase in motor activity, induces a reduction in movement, suggesting that the PPN recruits spinal circuits to halt locomotion (Takakusaki et al., 2016). The present ex vivo and in vivo experiments show that activation of PPN afferents induces inhibition of SPNs and therefore is capable of inhibiting the striatal output. Consistent with these findings, unilateral activation of PPN axons in the striatum in freely moving mice induced ipsilateral head rotations, in line with the optogenetic silencing of both populations of SPNs (Tecuapetla et al., 2014). In other words, excitatory afferents from the PPN/mesencephalic locomotor region are capable of suppressing striatal output by means of a feedforward inhibition mechanism. Together with the recently described functions of movement inhibition, our results situate 
the PPN as a key modulator of striatal activity. Furthermore, our findings reveal the PPN as the only known excitatory input capable of driving striatal inhibition without concurrent excitation of SPNs.

\section{References}

Albin RL, Young AB, Penney JB (1989) The functional anatomy of basal ganglia disorders. Trends Neurosci 12:366-375.

Assous M, Tepper JM (2019) Excitatory extrinsic afferents to striatal interneurons and interactions with striatal microcircuitry. Eur J Neurosci 49:593-603.

Assous M, Kaminer J, Shah F, Garg A, Koós T, Tepper JM (2017) Differential processing of thalamic information via distinct striatal interneuron circuits. Nat Commun 8:15860.

Assous M, Faust TW, Assini R, Shah F, Sidibe Y, Tepper JM (2018) Identification and characterization of a novel spontaneously active bursty GABAergic interneuron in the mouse striatum. J Neurosci 38:5688-5699.

Atallah BV, Bruns W, Carandini M, Scanziani M (2012) Parvalbuminexpressing interneurons linearly transform cortical responses to visual stimuli. Neuron 73:159-170.

Bradfield LA, Bertran-Gonzalez J, Chieng B, Balleine BW (2013) The thalamostriatal pathway and cholinergic control of goal-directed action: interlacing new with existing learning in the striatum. Neuron 79:153166.

Brill J, Mattis J, Deisseroth K, Huguenard JR (2016) LSPS/optogenetics to improve synaptic connectivity mapping: unmasking the role of basket cell-mediated feedforward inhibition. eNeuro 3:ENEURO.0142-15.2016.

Caggiano V, Leiras R, Goñi-Erro H, Masini D, Bellardita C, Bouvier J, Caldeira V, Fisone G, Kiehn O (2018) Midbrain circuits that set locomotor speed and gait selection. Nature 553:455-460.

Cruikshank SJ, Lewis TJ, Connors BW (2007) Synaptic basis for intense thalamocortical activation of feedforward inhibitory cells in neocortex. Nat Neurosci 10:462-468.

Dautan D, Huerta-Ocampo I, Witten IB, Deisseroth K, Bolam JP, Gerdjikov T, Mena-Segovia J (2014) A major external source of cholinergic innervation of the striatum and nucleus accumbens originates in the brainstem. J Neurosci 34:4509-4518.

Dautan D, Huerta-Ocampo I, Valencia M, Kondabolu K, Gerdjikov TV, Mena-Segovia J (2018) Cholinergic midbrain afferents modulate striatal circuits and shape encoding of action control. bioRxiv. Advance online publication. Retrieved August 8 2018. doi:10.1101/388223.

DeLong MR (1990) Primate models of movement disorders of basal ganglia origin. Trends Neurosci 13:281-285.

Ding JB, Guzman JN, Peterson JD, Goldberg JA, Surmeier DJ (2010) Thalamic gating of corticostriatal signaling by cholinergic interneurons. Neuron 67:294-307.

Doig NM, Magill PJ, Apicella P, Bolam JP, Sharott A (2014) Cortical and thalamic excitation mediate the multiphasic responses of striatal cholinergic interneurons to motivationally salient stimuli. J Neurosci 34:31013117.

English DF, Ibanez-Sandoval O, Stark E, Tecuapetla F, Buzsáki G, Deisseroth K, Tepper JM, Koós T (2011) GABAergic circuits mediate the reinforcement-related signals of striatal cholinergic interneurons. Nat Neurosci 15:123-130.

Faust TW, Assous M, Tepper JM, Koós T (2016) Neostriatal GABAergic interneurons mediate cholinergic inhibition of spiny projection neurons. J Neurosci 36:9505-9511.

Gabernet L, Jadhav SP, Feldman DE, Carandini M, Scanziani M (2005) Somatosensory integration controlled by dynamic thalamocortical feedforward inhibition. Neuron 48:315-327.

Galtieri DJ, Estep CM, Wokosin DL, Traynelis S, Surmeier DJ (2017) Pedunculopontine glutamatergic neurons control spike patterning in substantia nigra dopaminergic neurons. Elife 6:e30352.

Gittis AH, Nelson AB, Thwin MT, Palop JJ, Kreitzer AC (2010) Distinct roles of GABAergic interneurons in the regulation of striatal output pathways. J Neurosci 30:2223-2234.

Goldberg JA, Reynolds JN (2011) Spontaneous firing and evoked pauses in the tonically active cholinergic interneurons of the striatum. Neuroscience 198:27-43.

Ibáñez-Sandoval O, Tecuapetla F, Unal B, Shah F, Koós T, Tepper JM (2010) Electrophysiological and morphological characteristics and synaptic con- nectivity of tyrosine hydroxylase-expressing neurons in adult mouse striatum. J Neurosci 30:6999-7016.

Ibáñez-Sandoval O, Tecuapetla F, Unal B, Shah F, Koós T, Tepper JM (2011) A novel functionally distinct subtype of striatal neuropeptide $\mathrm{Y}$ interneuron. J Neurosci 31:16757-16769.

Isaacson JS, Scanziani M (2011) How inhibition shapes cortical activity. Neuron 72:231-243.

Josset N, Roussel M, Lemieux M, Lafrance-Zoubga D, Rastqar A, Bretzner F (2018) Distinct contributions of mesencephalic locomotor region nuclei to locomotor control in the freely behaving mouse. Curr Biol 28:884-901.e3.

Kawaguchi Y (1993) Physiological, morphological, and histochemical characterization of three classes of interneurons in rat neostriatum. J Neurosci 13:4908-4923.

Kemp JM, Powell TP (1971) The termination of fibres from the cerebral cortex and thalamus upon dendritic spines in the caudate nucleus: a study with the Golgi method. Philos Trans R Soc Lond B Biol Sci 262:429-439.

Klug JR, Engelhardt MD, Cadman CN, Li H, Smith JB, Ayala S, Williams EW, Hoffman H, Jin X (2018) Differential inputs to striatal cholinergic and parvalbumin interneurons imply functional distinctions. Elife 7:e35657.

Koós T, Tepper JM (1999) Inhibitory control of neostriatal projection neurons by GABAergic interneurons. Nat Neurosci 2:467-472.

Lee SH, Kwan AC, Zhang S, Phoumthipphavong V, Flannery JG, Masmanidis SC, Taniguchi H, Huang ZJ, Zhang F, Boyden ES, Deisseroth K, Dan Y (2012) Activation of specific interneurons improves V1 feature selectivity and visual perception. Nature 488:379-383.

Mallet N, Le Moine C, Charpier S, Gonon F (2005) Feedforward inhibition of projection neurons by fast-spiking GABA interneurons in the rat striatum in vivo. J Neurosci 25:3857-3869.

Martiros N, Burgess AA, Graybiel AM (2018) Inversely Active Striatal Projection Neurons and Interneurons Selectively Delimit Useful Behavioral Sequences. Curr Biol 28:560-573.e5.

Matsumoto N, Minamimoto T, Graybiel AM, Kimura M (2001) Neurons in the thalamic CM-pf complex supply striatal neurons with information about behaviorally significant sensory events. J Neurophysiol 85:960-976.

Mena-Segovia J, Bolam JP (2017) Rethinking the pedunculopontine nucleus: from cellular organization to function. Neuron 94:7-18.

Moyer JT, Halterman BL, Finkel LH, Wolf JA (2014) Lateral and feedforward inhibition suppress asynchronous activity in a large, biophysicallydetailed computational model of the striatal network. Front Comput Neurosci 8:152.

Nelson AB, Hammack N, Yang CF, Shah NM, Seal RP, Kreitzer AC (2014) Striatal cholinergic interneurons drive GABA release from dopamine terminals. Neuron 82:63-70.

Owen SF, Berke JD, Kreitzer AC (2018) Fast-spiking interneurons supply feedforward control of bursting, calcium, and plasticity for efficient learning. Cell 172:683-695.e15.

Pan WX, Mao T, Dudman JT (2010) Inputs to the dorsal striatum of the mouse reflect the parallel circuit architecture of the forebrain. Front Neuroanat 4:147.

Parthasarathy HB, Graybiel AM (1997) Cortically driven immediate-early gene expression reflects modular influence of sensorimotor cortex on identified striatal neurons in the squirrel monkey. J Neurosci 17:2477-2491.

Pouille F, Marin-Burgin A, Adesnik H, Atallah BV, Scanziani M (2009) Input normalization by global feedforward inhibition expands cortical dynamic range. Nat Neurosci 12:1577-1585.

Ramanathan S, Hanley JJ, Deniau JM, Bolam JP (2002) Synaptic convergence of motor and somatosensory cortical afferents onto GABAergic interneurons in the rat striatum. J Neurosci 22:8158-8169.

Ros H, Magill PJ, Moss J, Bolam JP, Mena-Segovia J (2010) Distinct types of non-cholinergic pedunculopontine neurons are differentially modulated during global brain states. Neuroscience 170:78-91.

Roseberry TK, Lee AM, Lalive AL, Wilbrecht L, Bonci A, Kreitzer AC (2016) Cell-type-specific control of brainstem locomotor circuits by basal ganglia. Cell 164:526-537.

Saunders A, Huang KW, Sabatini BL (2016) Globus pallidus externus neurons expressing parvalbumin interconnect the subthalamic nucleus and striatal interneurons. PLoS One 11:e0149798.

Schulz JM, Reynolds JN (2013) Pause and rebound: sensory control of cholinergic signaling in the striatum. Trends Neurosci 36:41-50.

Silberberg G, Bolam JP (2015) Local and afferent synaptic pathways in the striatal microcircuitry. Curr Opin Neurobiol 33:182-187. 
Smith Y, Raju DV, Pare JF, Sidibe M (2004) The thalamostriatal system: a highly specific network of the basal ganglia circuitry. Trends Neurosci 27:520-527.

Smith Y, Galvan A, Ellender TJ, Doig N, Villalba RM, Huerta-Ocampo I, Wichmann T, Bolam JP (2014) The thalamostriatal system in normal and diseased states. Front Syst Neurosci 8:5.

Straub C, Saulnier JL, Bègue A, Feng DD, Huang KW, Sabatini BL (2016) Principles of synaptic organization of GABAergic interneurons in the striatum. Neuron 92:84-92.

Szydlowski SN, Pollak Dorocic I, Planert H, Carlén M, Meletis K, Silberberg G (2013) Target selectivity of feedforward inhibition by striatal fast-spiking interneurons. J Neurosci 33:1678-1683.

Takakusaki K, Chiba R, Nozu T, Okumura T (2016) Brainstem control of locomotion and muscle tone with special reference to the role of the mesopontine tegmentum and medullary reticulospinal systems. J Neural Transm (Vienna) 123:695-729.

Tecuapetla F, Matias S, Dugue GP, Mainen ZF, Costa RM (2014) Balanced activity in basal ganglia projection pathways is critical for contraversive movements. Nat Commun 5:4315.

Tepper JM, Koos T, Ibanez-Sandoval O, Tecuapetla F, Faust TW, Assous M (2018) Heterogeneity and Diversity of Striatal GABAergic Interneurons: Update 2018. Front Neuroanat 12:91.

Tepper JM, Wilson CJ, Koos T (2008) Feedforward and feedback inhibition in neostriatal GABAergic spiny neurons. Brain Res Rev 58:272-281.

Wall NR, De La Parra M, Callaway EM, Kreitzer AC (2013) Differential innervation of direct- and indirect-pathway striatal projection neurons. Neuron 79:347-360.

Wang HL, Morales M (2009) Pedunculopontine and laterodorsal tegmental nuclei contain distinct populations of cholinergic, glutamatergic and GABAergic neurons in the rat. Eur J Neurosci 29:340-358.

Xenias HS, Ibáñez-Sandoval O, Koós T, Tepper JM (2015) Are striatal tyrosine hydroxylase interneurons dopaminergic? J Neurosci 35:6584-6599. 